\title{
A self-aware paradigm for autonomous architectural systems within the Internet of Things
}

\author{
Ahmed Elsherif a , Dina Mandour ${ }^{\mathrm{b}}$, May A.Malek Alic ${ }^{\mathrm{c}}$, Mohamed Sedky \\ ${ }^{a}$ Interior Design and Furniture Department, Pharos University in Alexandria, \\ Alexandria, Egypt; ${ }^{b}$ Interior Architecture Department, Alexandria University, \\ Alexandria, Egypt; ' Interior Architecture Department, Alexandria University, \\ Alexandria, Egypt; ${ }^{d}$ School of Computing (Networks, Cybercrime \& Embedded \\ Systems), Staffordshire University, Stafford, United Kingdom \\ a'Demonstrator, Faculty of Arts and Design (ahmed_essam_90@hotmail.com) \\ bProfessor, Faculty of Fine Arts (dinamandour@yahoo.com) \\ ${ }^{\mathrm{c} A s s i s t a n t ~ p r o f e s s o r, ~ F a c u l t y ~ o f ~ F i n e ~ A r t s ~(m a y m a l e k @ ~ h o t m a i l . c o m) ~}$ \\ ${ }^{\mathrm{d}}$ Assistant professor, Faculty of Computing, Engineering and Sciences \\ (m.h.sedky@staffs.ac.uk)
}




\section{A self-aware paradigm for autonomous architectural systems within the Internet of Things}

This research explores a newfangled approach for the notion that architecture is no longer concerned with the organization of space and matter, but rather a system of systems with self-organizational behavior and progressively complex programmatic interventions. Such system represents an Internet of Things (IoT) paradigm that has the capacity to self-organize a pre-defined architecture acclimating dynamically to the demands of the environment through a process of self-awareness and re-configurability.

This paper is an attempt to develop spaces that bring computation into the physical world by introducing artificial intelligence into building systems so as to communicate, exchange information, and allow right responses and decisions within a sustainable manner. Environments with embedded computational systems and adaptive reconfiguration behavior will be precisely studied.

With an intensive focus on smart, self automated architecture that has embraced kinetic motion as an approach for environmental adaptation and responsiveness. This study addresses the networked organization of sensory systems that incorporate computational platforms in relation to user's desires, where architecture turns into an interactive intermediary between human and computation.

Correspondingly, the authors are particularly aiming to propose a conceptual framework for delivering a smart autonomous system at the architectural scale which is capable of reconfiguring and interacting constantly in real time.

Thus the IoT will foster a rapid development in intelligent systems which would directly introduce an advanced technological leap forward in accommodating a new way of demonstrating human-computer interaction within a novel architectural design approach.

Keywords: word; Internet of Things, adaptive reconfiguration, autonomous architectural systems, artificial intelligence, responsive behavior, embedded computation

\section{Introduction}

Recent studies have emphasized the technological potentials for the Internet of Things (IoT) as a communication strategy that conceives a smart autonomous paradigm, in 
which the physical objects of everyday life would be consolidated with microcontrollers and embedded sensors for digital communication. This makes the IoT able to combine diversified parameters into everyday objects and activities so as to communicate with one another and users, and respond to various environmental stimuli through the internet. (Höller et al., 2014)

Such objects confer novel computing implementations, and are widely recognized for introducing a comprehensive framework of networked physical objects recognized nowadays as the Internet of Things (IoT), (Bessis \& Dobre, 2014) that considers ubiquitous presence in the environment of a diversity of objects which through wireless and wired connections and distinct addressing platforms are able to communicate with each other and cooperate with other things to create novel applications and services, achieving common objectives. (Vermesan \& Friess, 2013)

The particular hypothesis introduced derives from recent developments in "Ubiquitous Computing" as a functional integration of computer sciences and advanced design streams. Through a computational platform, architectural spaces jointly engage into the context of everyday matters as an ideology of human-computer interaction. This generates architectural spaces which embed computation into the physical world within a smart environment encompassing autonomous configurations and communication strategies. Correspondingly, the environment can be comprehended as an emergent space of re-configurable organization of systems, reacting continually within a direct engagement and interaction where users become participants within a behavioral framework based on unpretentious principles of contextual parameters and complex symbiotic relationships of communication through people-to-people, people-tobuilding; and building-to- building. (Fox \& Kemp, 2009) 
In this framework, re-configurable architectural systems require a standpoint to design that incorporates the requirements of the present with the potentials to adjust to considerably variable needs in the future. This particular approach is based on supporting participants and designers, who can mainly recognize their own functions and circumstances, with the capacity to make convenient configurations when required, and discover newfangled models of living and participating within their built environment, through models of interactions, and data-intensive computing as an adaptive network of time-based systems (Long et al., 2008)

Correspondingly, data-intensive computing can be deliberated on the basis for a novel paradigm of infrastructures permitting to constantly storing these data for participation and analyzing through the Internet and developed technologies of real-time simulation, and embedded sensory systems that transform our daily environmental matters into smart ones capable to comprehend and respond to their surroundings.

Thereby the authors attempt to enable an ideological alteration in the cognition of the inhabited space through proposing an autonomous model of self-aware system, supporting the architectural space within hybrid control networks, and controlled through the Internet of Things.

\section{Literature review-Theoretical background}

Recent developments have started to initiate a move from a mechanical ideology of versatility to a biological one. The commonness of the organic paradigm is starting to change the conceptual ideology that we enforce with a view to comprehend our environment and, accordingly, design within its surroundings. (Bouzanjani, 2013)

Starting with Gordon Pask at the 1960's, with a distinctive progress toward comprehending and recognizing the field of cybernetics within the architectural framework by developing his well-known theories. Pask who later participated with 
diverse architects in the 1970s and 1980s, developed a "Conversation Theory," a specifically consistent and potentially the most prolific theory of interaction and communication comprising human-to-human, human-to-machine and machine-tomachine configurations in a common framework. (Haque, 2007) The theory served as the premise of the implied processes implicated in complex and intelligent learning methodologies through humans and machines that enable learners to realize commonsensical comprehension of the complex pragmatic processes for a multilevel system through diverse models of communication within participants, transferring what they have learned in a continuous discourse. (Boyd, 2008)

The concept has been kept on being developed through the work of Nicholas Negroponte and the Architecture Machine Group at the MIT from the late 1960's to the mid 1970`s. Negroponte presented the implementation of computers in architectural systems and embraced their incorporation in spatial structures and architectural spaces at the "Software" exhibition through a responsive model inscribed in a plexiglass case, embedded with an intelligently-controlled environment of small cubic blocks and a number of gerbils changing the blocks' arrangement while a programmed robotic arm autonomously lay out the blocks in an order trying to follow the gerbil's movements. (Yan, 2015)

The objectives were to develop a responsive paradigm within the architectural platform in addition to creating an adaptive environment that opens up the possibilities to interact and respond to the requirements and desires of users. It is a fundamental model of a cybernetic reactive cycle placed in a building environment in which the current output is influenced according to a particular input. (Towfiq, 2012) Negroponte's responsive architecture is well-considered as a notion of smartness and versatile response. Negroponte believes that comprising artificial intelligence with 
architectural environments is crucial to delivering an adaptive, responsive architecture which have the capacity for reacting autonomously according to assorted needs.

And through the 1980s and 1990s, a groundbreaking advancement started within the field of software engineering and computer science which has delivered the notion of "intelligent environments" as a framework to investigate spaces with embedded systems and communication strategies, generating spaces that bring computation into the physical world as a central control system for everything. (Fox, 2014)

Reaching 2013, research scientists at MIT's Computer Science and Artificial Intelligence Laboratory has long conceived for an upcoming ideology mobile, modular robotic system renowned as M-Blocks -a self-assembling robotic approach for futuristic constructions. The kinetic prototype robotic cube is characterized by the absence of outward movable appliances, which is capable of scrolling and climbing over, around and jump onto each other utilizing an internal flywheel so that the blocks can reconfigure themselves in novel adjustments. (Cruz, 2013)

\section{Problems with conventional architectural streams}

Throughout history, we can obviously realize the miscellaneous approaches representing conventional architecture are highly restricted by the lack of adaptive responses, flexibility and dynamism, where spatial configurations are static and can be barely capable of accommodating the range of contingencies that depict daily life as shifts in environmental circumstances, changes in the behavior and needs of people. (Pan \& Jeng, 2008)

Our buildings are inconsistent with the connotation of change. Constant utilities, inflexible materials, static infrastructure and lack of real-time adjustments impede our ability to affect our surroundings. Architects have no approach to access the future user requirements in the design process while there are constant demands for adding 
versatility and dynamism within our built environments ranging from programmatic and site-context response to spatial dynamics. (Long et al., 2008)

It can be widely-recognized the importance of addressing responsiveness within accustomed architectural spaces as they are not depicted by their capacity to adapt continuously in real time and stay aware with the continuously assorted requirements of various users, where the general state of the space may be optimized for accommodating various users. However, it is not adjusted to alter its physical shape adapting with their diverse particular functions within a time-based system.

And hence, our current study mainly addresses problems dealing with the continuous change in functions within the interior spaces for universities comprising numerous variables ranging from number of students attending a particular course, time schedule for professors, diverse sorts of programs integrating courses, lectures, workshops and seminars where each require a particular organization for the architectural space encompassing the precise program held.

\section{The Internet of Things as a smart model for self-aware configurations}

The paper primarily focuses on introducing three diverse approaches for reconfiguring an architectural space -for a smart campus- through the Internet of Things as an autonomous paradigm for anticipating and receiving parameters of particular inputs within a relevant context of students' interaction with university programs, analyzing and processing data, and adjusting its mode through augmenting the human functionality within a self-aware framework as described in figure 1 . The particular methodologies are based on computational and physical strategies of adaptive configuration that can self-regulate, respond to changes and support both communication and interaction, impacting the way students interact with their space, through variable adjustments in its geometry to accommodate diverse automated 
lecture-seminar halls, workshop zones, and workspace within a single space that can physically reconfigure to face assorted changing needs.

And hence, the described physical space would accommodate a new way of manifesting human-computer interaction in architectural design systems, which communicate, exchange information through direct engagement integrating the upsides of automation with responsive systems and smart configurations, where the objects within the smart campus might physically exist only when fundamentally required and transform when they are no longer practically needed.

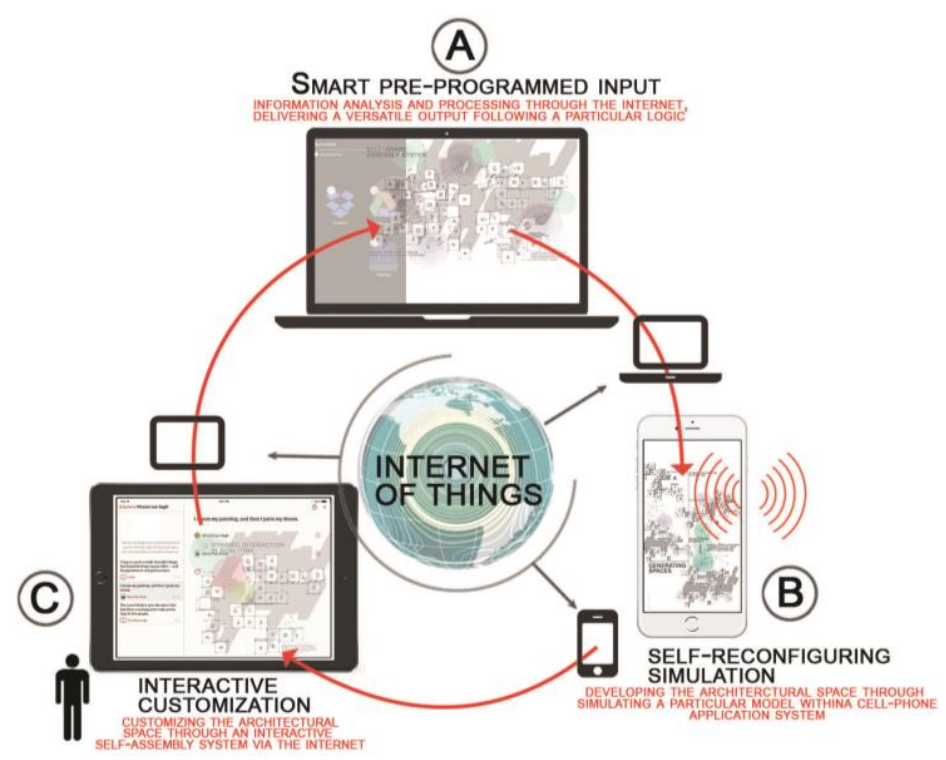

Figure 1. Systems of space-configuration using the Internet of Things

\section{Smart-controlled constructional set}

The paper introduces an autonomous behavior for an architectural system controlled through the Internet of Things that enables a programmatic, extensive and dynamic interaction in real time, trying to explore the dynamics of architectural space by rethinking architecture beyond conventional static and single-function spatial design. It proposes a programmed control system through the internet for a summer school's program encompassing a number of workshops, seminars and courses, each customized 
for a particular architectural space organization which is directly linked to the number of registering students. The authors would propose three sequential platforms for autonomous reconfigurations through the Internet of Things.

\subsection{Smart pre-programmed input}

Custom algorithms and programming scripts have been utilized developing a conceptual online program which is directly linked to an experimental customized prototype via the internet emphasizing its autonomy within a versatile kinetic notion of responsiveness.

The experimental approach is based on delivering diverse programmed motions and rotations as an output following a particular input through the internet as in figure 2

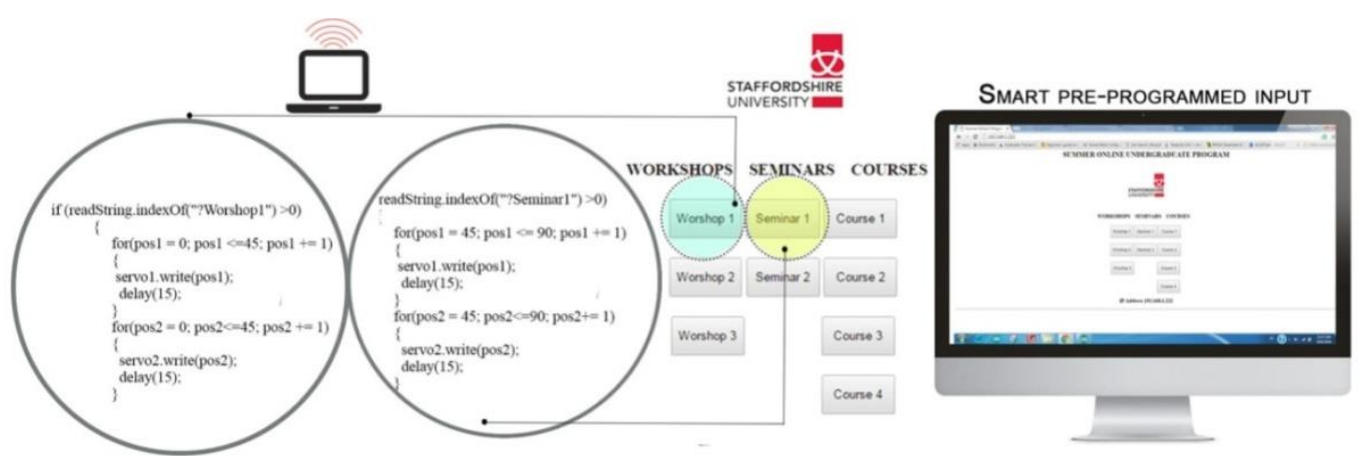

Figure 2. Part of script for rotational response following particularly-analyzed data

\subsubsection{Self-reconfiguring discourse for the autonomous constructional set}

On registering a particular program through a specific number of students, the autonomous system starts to aggregate through a self-assembly process following a particular algorithm-driven design logic which exhibits methods of self-awareness and versatile configuration with respect to constantly changing needs within a notion of selfassembly which is described in figure 3 .

\subsection{Self-reconfiguring simulation}

Using the Internet of Things, users have been capable to directly choose their own architectural space, where its elements are complexly layered and continuously 
differentiated within a time-based system through a stimuli-responsive behavior. The particular approach is based on simulating a specific model within a cell-phone application system. Depending on the continuous change in needs, students choose from a number of customized architectural proposals as in figure 4.

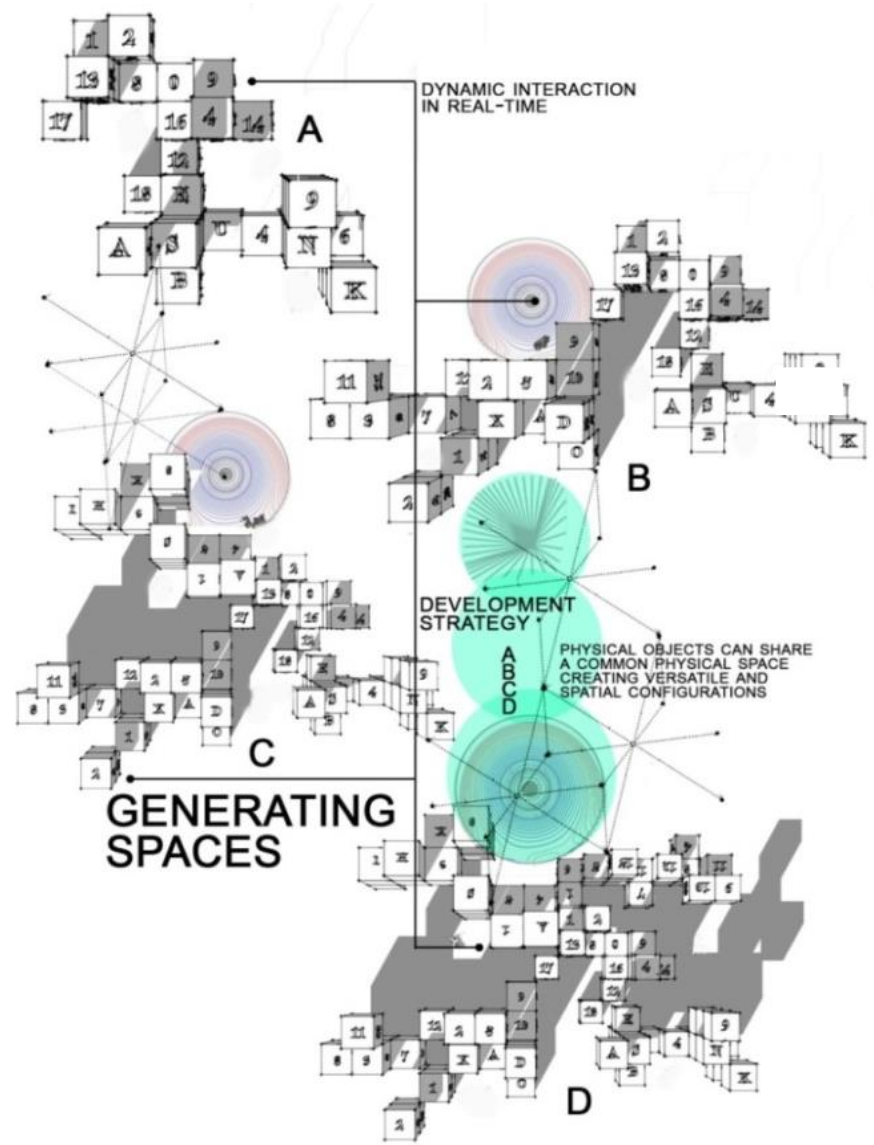

Figure 3. Self-reconfiguring system comprising smart aggregation process
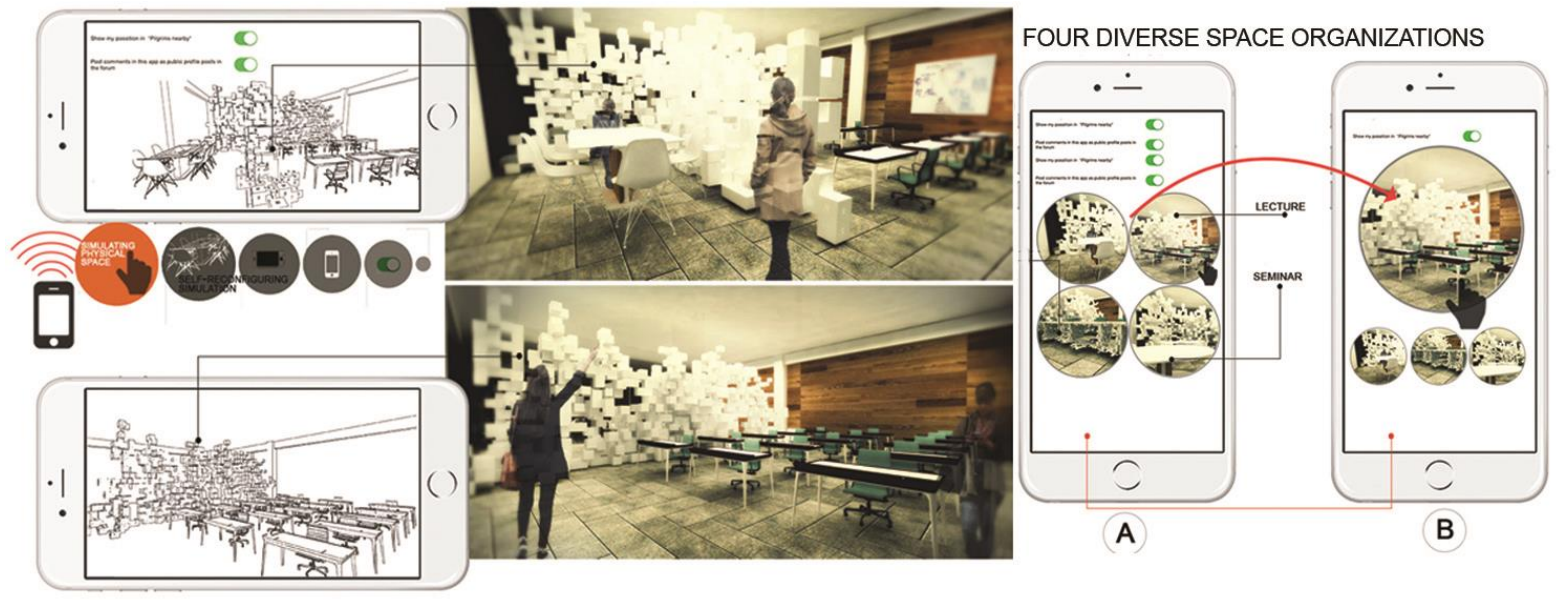

Figure 4. Self-reconfiguring simulation for physical space via cell-phone application system 


\subsubsection{Self-reconfiguring furniture's framework}

Through diverse aggregation modules, the autonomous system demonstrates high capacities in achieving a transformational context within the scale of furniture units according to the required function as described in figure 5 .

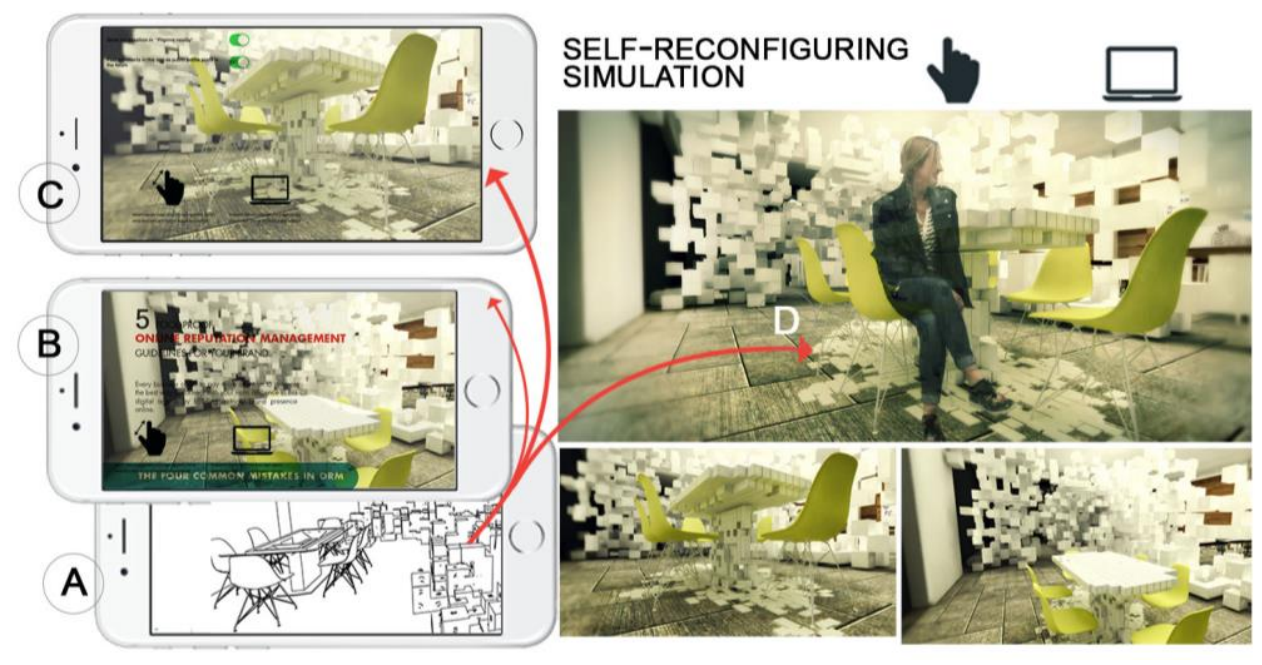

Figure 5. Aggregation process for smart sets developing self-reconfiguring furniture corresponding to data-driven inputs from smart-phone

\subsection{Interactive customization}

The interactive customization system is based on computational and physical strategies of adaptation, where it organizes the architectural space in a building-up process creating a number of zones appropriate with the particular program required within an intelligent constructional approach as in figure 6.

The physical space is highly customized generating diverse zones which are variable in their number and space organization following particular inputs received.

\section{Advantages for the proposed autonomous self-reconfiguring system}

The system is directly capable of delivering three diverse modes of real-time settlings and customized alternatives which can highly adapt to diverse users' demands for space organization within a notion of smart campus through the Internet of Things. The advantages of the autonous system can be summarized as 


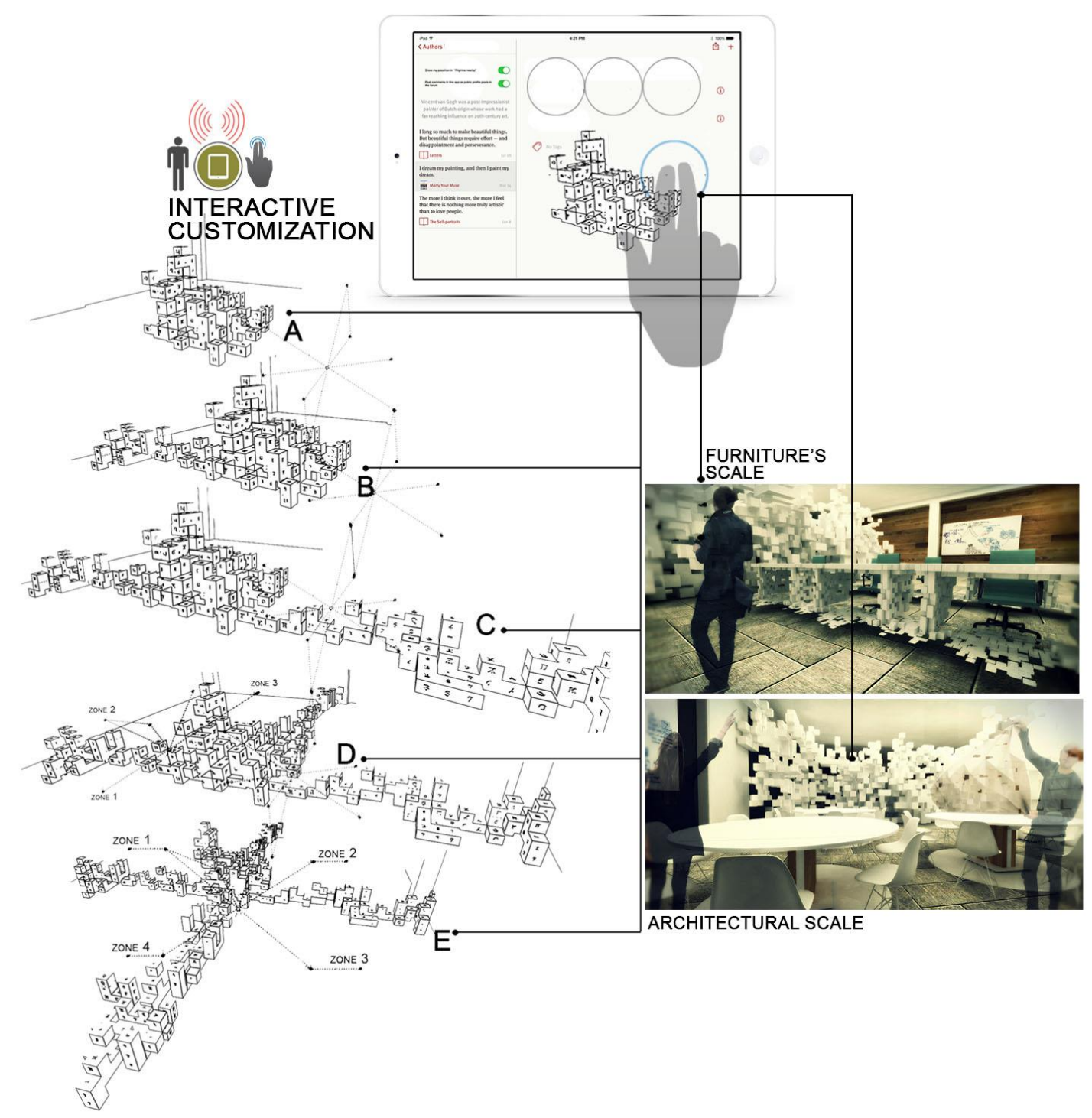

Figure 6. Intelligently-customized aggregation process

- The proposed sets are modular units which can undergo transformations from the architectural scale to the furniture's ones following a particular preprogrammed input, self reconfiguring simulation, or interactive customization behavior.

- The system embraces the Internet of Things with a view to alter the way people interact with space within their smart campus regarding the context of optimization, as it accommodates particular user requirements through being capable of responding to an optimized framework to target a specific sapaceconfiguration. 
- The smart kinetic notion within the autonomous system is based on an algorithm-driven design strategy which is inherently adaptive with regard to diverse objectives and platforms, in addition to its capacity to be applied through various architectural frameworks.

\section{Challenges facing the relevance of autonomous systems wide spreading within the architectural context}

It can be widely recognized the robust correlation of adaptive responses and interaction within architecture during the preliminary constructional phases, taking into account its wide spreading as an advanced framework in recent years, we highly face sort of challenges comprising the integration of various embedded systems within a machine learning framework that delivers algorithm driven-design configurations for generating architectural spaces following diverse particular inputs and variables.

We have further challenges concerning the economical feasibility and costeffectiveness for the wide propagation needed to bring about out the notion of selfawareness within the autonomous robotic systems addressing real changes during daily behavioral cycles within a sustainable manner, as the hardware, software, and fabrication domains are cost- exorbitant, in addition to the necessity for software simulations rather than physical experimentations. However, nowadays a number of diverse factors and demands have substantially accelerated the ease of responsive prototyping as well as full-scale implementation. Much of this feasibility have begun from various approaches, technologies, and frameworks that have been expanded upon other intelligently-driven fields paralleling architecture, such as smart home technologies, which have highly participated in developing a number of responsive systems within the labor market. This propagation in smart notions has contributed in 
reducing the hardware and software's price, which have led to higher degrees of environmental coherence and performance relevant to the appropriate resources.

In terms of structural performance, the mobile self-reconfiguring system needs higher demands to develop newfangled structural potentials other than the accustomed ones, generating an adaptive and mobile architectural space which can respond to diverse inputs while holding its structure up against physical forces.

\section{Conclusion}

The paper particularly emphasizes the correlation between technology and architecture, in which advances in one field empower or demand changes in the other, as introducing computer science discipline within the architectural context has altered the way people interact with their built environment.

The study introduces the Internet of Things as a smart control discourse within the architectural space through three models of computational platforms which have been utilized to develop autonomous configurations among three-dimensional intelligent geometries.

The transformation of the architectural paradigm through the Internet of Things is driven by the potentials to embed intelligence into building's systems and connect them in real-time, where moving physical objects can share a common physical space creating versatile and spatial adjustments, encompassing together physical world of objects, humans and virtual conversation and interactions.

Progresses in ubiquitous and pervasive computing are opening up new scopes for developing a newfangled vision of a smart, responsive architecture that adapt efficiently to complex site and programmatic necessities tending to today's dynamic, flexible and constantly changing needs. 


\section{References}

Bessis, N., \& Dobre, C. (Eds.). (2014). Big data and Internet of things: A Roadmap for smart environments. Switzerland: Springer International Publishing AG

Bouzanjani, B. F. (2013). ALLOPLASTIC ARCHITECTURE: THE DESIGN OF AN INTERACTIVE. Retrieved from http://papers.cumincad.org/data/works/att/acadia13_129.content.pdf

Boyd, G. M. (2008). The human centrality of conversational learning. Handbook of Conversation Design for Instructional Applications, 49.

Cruz, J. L. G. (2013, October 6). MIT researchers propose self-assembling robots as future of construction. Retrieved June 24, 2016, from archdaily,http://www.archdaily.com/435250/mit-researchers-propose-selfassembling-robots-as-future-of-construction

Fox, M. (2014). (Catching up with the past: A small contribution to a long history of interactive environments. FOOTPRINT, 4(1) 18-5. Retrieved from http://footprint.tudelft.nl/index.php/footprint/article/view/716/893)

Fox, M., \& Kemp, M. (2009). Interactive architecture. New York: Princeton Architectural Press.

Haque, U. (2007). The architectural relevance of Gordon Pask. Architectural Design, 77(4), 54-61. doi:10.1002/ad.487.

Höller, J., Tsiatsis, V., Fikouras, I., Avesand, S., \& IVONNE, P. (2014). From machineto-machine to the Internet of things: Introduction to a new age of intelligence. Amsterdam: Academic Press.

Long, J., Magnolfi, J., \& Maassen, L. (2008) Always building: the programmable environment. Retrieved from http://www.hermanmiller.com/content/dam/hermanmiller/documents/always_bu ilding/always_building.pdf

Pan, C.A., \& Jeng, T. (2008) Exploring Sensing-based Kinetic Design for Responsive Architecture. Retrieved from http://papers.cumincad.org/data/works/att/caadria2008_35_session4a_285.conte nt.pdf

Towfiq. (2012). Daniel Grünkranz: Towards a Phenomenology of responsive architecture: Intelligent technologies and their influence on the experience of space. Retrieved June 24, 2016, from http://formsociety.com/2012/07/daniel- 
grunkranz-towards-a-phenomenology-of-responsive-architecture-intelligenttechnologies-and-their-influence-on-the-experience-of-space/

Vermesan, O., \& Friess, P. (Eds.). (2013). Internet of things: Converging technologies for smart environments and integrated ecosystems. Aalborg: River Publishers.

Yan, J. (2015, November ). Unpredictable Cybernetic zoo - MAT200aF2015. Retrieved from http://cargocollective.com/200AF2015/Unpredictable-Cybernetic-Zoo 